\title{
Assessment of a new synbiotic preparation in healthy volunteers: survival, persistence of probiotic strains and its effect on the indigenous flora
}

\author{
Lorenzo Morelli*†1, Daniela Zonenschain $^{\dagger 1}$, Maria Luisa Callegari ${ }^{\dagger 2}$, \\ Enzo Grossi ${ }^{\dagger 3}$, Federico Maisano ${ }^{\dagger 3}$ and Michele Fusillo ${ }^{\dagger 3}$
} \begin{abstract}
Cremona-Italy and ${ }^{3}$ Medical Department, Bracco SpA, Via Folli 50, Milan Italy
Email: Lorenzo Morelli* - lorenzo.morelli@unicatt.it; Daniela Zonenschain - daniz1971@libero.it; Maria Luisa Callegari - marialuisa.callegari@unicatt.it; Enzo Grossi - enzo.grossi@bracco.com; Federico Maisano - federico.maisano@bracco.com; Michele Fusillo - michele.fusillo@bracco.com

* Corresponding author †Equal contributors
\end{abstract}

Address: ${ }^{1}$ Istituto di Microbiologia - UCSC, Via Emilia Parmense 84 Piacenza-Italy, ${ }^{2}$ Centro Ricerche Biotecnologiche UCSC, Via Milano 24 -

Published: 09 October 2003

Nutrition Journal 2003, 2:11
Received: 31 July 2003

Accepted: 09 October 2003

This article is available from: http://www.nutritionj.com/content/2/I/II

(c) 2003 Morelli et al; licensee BioMed Central Ltd. This is an Open Access article: verbatim copying and redistribution of this article are permitted in all media for any purpose, provided this notice is preserved along with the article's original URL.

\begin{abstract}
Background: Use of synbiotic preparations as dietary supplement is believed to be a valid approach to restore and maintain colonic microflora. However, only few papers have been published on the assessment of these food supplements and none of them have used molecular biology techniques to evaluate the effects of the probiotic components.

Methods: Twelve healthy volunteers were recruited. Faecal samples were taken before and at various time points during the administration period and at day 3 in the post-treatment period. Stool culture were performed and amplified ribosomal DNA restriction analysis was used to detect $L$. paracasei, the major bacterial component of the synbiotic products.

Results: An increase of at least I log of $L$. paracasei-like bacteria was observed in all subjects. An increase of as much as 3 log was seen in subjects who had a low number of $L$. paracasei-like lactobacilli at the baseline. The counts of $L$. paracasei-like lactobacilli were found to persist for at least 3 days after discontinuation of intake in healthy volunteers in 7 subjects. Genetic analysis showed that the maiority of vancomicin insensitive lactobacilli were real $L$. paracasei, as the strains administered with the tested product.

Conclusion: This study has shown that the strains of $L$ paracasei administered with a synbiotic dietary supplement are able to survive through the gastrointestinal tract and to persist for at least a few days. It was also shown the efficacy of a synbiotic preparation to positively affect the microflora of healthy volunteers.
\end{abstract}

\section{Background}

For a long time colonic microflora has been considered to play an important role in the maintenance of the health and well-being of the host [1]. In addition to promote normal gastrointestinal functions and protecting against pathogenic bacteria, the microflora exerts beneficial effects on systemic metabolism and immune system [2]. 
The ability to control the growth and the pathogenic potential of these bacteria depends on the proper function of the microflora [3].

Imbalance in the colonic microflora with relative predominance of aggressive bacteria and insufficient concentration of protective species has been associated with colonic inflammation $[4,5]$ and pouchitis[6].

Intake of probiotics (living micro-organisms), prebiotics (non-digestible oligosaccharides) and synbiotics (mixture of probiotics and prebiotics) has been demonstrated to modify the composition of the microflora, restore the microbial balance and therefore have the potential to provide health benefits [7-9]. However it has only been during the last few years that well designed clinical studies have provided clear evidence of health promoting effects, such as prevention of antibiotic-associated diarrhoea [10], treatment of acute diarrhoea[11], inflammatory bowel disease[12], eradication of $C$ difficile infection[13] and enhancement of intestinal immunity $[14,15]$.

The current state of evidence suggests that probiotic effects are strain-specific and even strains belonging to the same species may have marked or no probiotic effect [16].

As a result, in recent years there has been an increasing demand to select, by means of in vitro and in vivo tests, new strains with potential superior probiotic effects [17]. There is a general consensus that probiotic strains should be of human origin, as these bacteria have a greater chance of competing with resident bacteria, and of becoming numerically predominant after short intake and to persist in the colonic environment for some time after discontinuation of use.

Prebiotic substances are non digestible food ingredients which could be fermented by selected groups of beneficial bacteria; their positive influence on intestinal flora has been assed by a number of studies (for a review see [18]) The use of probiotic strains together with prebiotic substances will provide a combined effect, named "synbiotic" [19].

A large number of new lactobacilli strains have been previously isolated from faecal samples of newborns [20]; they were identified, by means of genetic analysis, as naturally persisting in the same subjects for several following days [20]. Following phenotypic characterisation and in vitro evaluation, three new lactobacilli strains ( $L$. paracasei strain B 21060, L. paracasei B21070 and L. gasseri strain B21090) have finally selected in view of their use as probiotics.
A synbiotic formulation, consisting of a mixture of the above selected strains, oligosaccharides as prebiotic ingredients, glutamin, vitamin B6 and zinc, has been developed. The rationale of this formulation is to exploit a complementary probiotic action resulting from the different intrinsic properties of each individual strain and the promotion of bifidobacterial growth due to oligosaccharides. This formulation has been here assessed in a nutritional trial aimed at evaluating the ability of the selected strains to survive, grow and persist along the gastrointestinal tract and its efficacy and safety in various gastrointestinal disorders when administered in the final pharmaceutical formulation, in order to follow the recently issued $\mathrm{FAO} / \mathrm{WHO}$ guidelines [16].

The primary aim of this study was to evaluate the ability of the probiotic strains delivered by the synbiotic preparation to survive following passage through the gastrointestinal tract and to persist in the stools after discontinuation of the intake in healthy volunteers.

The secondary aim was to evaluate the effects of the synbiotic formulation on some members of the indigenous flora.

\section{Methods \\ Subjects}

Twelve healthy volunteers participated in this study. Eligible participants were of both sexes and aged 24-48 years. Subjects were considered healthy on entry into the study if they did not have a history of chronic gastrointestinal diseases including chronic constipation and any episode of diarrhoea ( $>3$ bowel movements/day for 3 consecutive days) during the last month and did not present any current sign or symptom of gastrointestinal disorder or infection. Individuals were not included in the study if they were pregnant or breast-feeding, had a history of diabetes or had received antibiotics over the last 3 months before admission.

Subjects taking probiotic preparations including fermented milk had to discontinue the intake at least 2 weeks before entry into the study. Standard yoghurt, containing Lactobacillus bulgaricus and/or Streptococcus thermophilus only, was not prohibited. Before entry, all participants were screened medically for their suitability for the study.

Each subject signed an informed consent after he/she had been made fully aware of the purpose of the study.

\section{Synbiotic administration}

For the present study we used a new synbiotic preparation containing a combination of viable freeze-dried new lactobacilli strains of human origin with prebiotics 
(inuline, oligosaccharides), glutamine, zinc and vitamin B6.

The product was available as a powder and dispensed in 6-g bag. Each bag contained $5 \times 10^{9}$ of both $L$. paracasei strain B21060 and strain B21070 and $0.5 \times 10^{9}$ of L. gasseri strain B 21090 [Flortec, Bracco SpA, Milan]. Each subject was instructed to take one bag three times a day (before breakfast, lunch and dinner) for 15 days. The content of the powder had to be dissolved in $50 \mathrm{ml}$ of water before oral intake.

Strains are deposited at the Collection Nationale de Cultures de Microorganismes, Institute Pasteur (Paris).

\section{Study procedures}

The study had three periods: 7-day screening and baseline period (day-7 till day 0), 15-day intake period (day 1-day $15)$ and 3-day post-treatment period (day +1 -day +3$)$ Subjects' medical history, physical examination, and routine laboratory tests were taken at day-7. Faecal samples were collected at day- 7 and day 0 .

During the administration period subjects returned to deliver stool samples collected at day 5, 10 and 15, a general daily questionnaire on daily well-being, stool consistency and frequency and verification of compliance to the study procedures. Information on tolerability and possible adverse events was recorded at each visit.

In the post-treatment period subjects returned to deliver stool samples collected at day +3 . The faecal samples were collected in sterile disposables with $9 \mathrm{ml}$ of AMIES liquid (Difco, Detroit, Michigan), stored at $4-8^{\circ} \mathrm{C}$ and delivered to the Department of Microbiology within 12 hours after collection.

\section{Microbiological analysis of faeces}

Processing of samples occurred within 12 hours after collection.

Weighted samples (about $1 \mathrm{~g}$ ) were homogenised for $30 \mathrm{~s}$ in a stomacher (Stomacher 400, Seward, London, England) before dilution in a pre-reduced brain hearth infusion broth and cultivation on the appropriate selective media.

Appropriate dilutions were plated using Rogosa Acetate agar (Difco) and Rogosa Acetate agar (Difco) added with $12 \mu \mathrm{g} / \mathrm{ml}$ of vancomycin (Sigma) to enumerate total Lactobacillus spp. and vancomicyn insensitive lactobacilli (i.e. L. paracasei group, including L. paracasei, L. casei and L. rhamnosus), respectively.
Bifidobacterium strains were enumerated using TPY agar added with $12 \mu \mathrm{g} / \mathrm{ml}$ of nalidixic acid (Sigma, St. Louis, USA), while enterobacteria were counted on VRBA, enterocci on SB agar and $\mathrm{Cl}$. perfringens was counted on Clostridium perfrigens agar base.

All plates for lactobacilli were incubated for $48 \mathrm{hrs}$ at $37^{\circ} \mathrm{C}$ in anaerobic jars (GasPak, BBL, Coskeysville, MD, USA), while the incubation for clostridia was extended to 3 days. Enterocci were incubated in aerobic conditions for 24 hours and enterobacteriaceae for 12 hours.

\section{Genetic identification of $\mathrm{L}$. paracasei}

PCR-ARDRA (amplified ribosomal DNA restriction analysis) was performed to identify species among the vancomycin-insensitive Lactobacillus colonies (L. paracasei group). We used a set of four enzymes and five primers to amplify the 16S-rDNA sequences of the tested lactobacilli. This is a reliable and rapid method to recognise $L$. paracasei strains from L. casei and L. rhamnosus [21].

L. gasseri was not sought due to the lack of a selective medium with antibiotics able to reduce the number of CFUs to be checked by means of genetic analysis.

\section{Study endpoints}

In the planning of the study the ability to survive passage through the gastrointestinal tract was defined as successful if an increase of least one log in the counts of $L$. paracasei group was observed in the stool sample at the end of treatment compared to baseline. Persistence was considered adequate if the concentration in the faecal sample after a 3-day discontinuation of intake was equal or only slightly decreased $(\max 1 \log )$ compared to end of treatment.

In addition, an increase of total lactobacilli and Bifidobacterium and a decrease of Enterobacteriacee, enterococci and clostridium in the stool sample were considered as potential beneficial effects.

\section{Results}

Microbiological assessment

All the 12 subjects ( $5 \mathrm{M}, 7 \mathrm{~F}$, range 24 to 48 years old) completed the study. The preparation was tolerated and accepted very well by each participant. No adverse effect was registered during the study.

The microbiology examination of faecal samples showed an increase of at least $1 \log$ of $L$. paracasei group in all subjects over the intake period. The increase in the counts was rapid and mostly evident after 5 day-administration. Six subjects who had a low number of counts of $L$. casei group at the baseline had in increase of even 3 log during the intake (Table 1). 
Table I: Changes in the counts of lactobacilli and bifidobacteria following intake of synbiotic preparation. Individual values. Values are in log of CFU/g of faeces

\begin{tabular}{|c|c|c|c|c|c|c|c|c|c|c|c|c|}
\hline Time-Bacteria/Vol. no. & I & 2 & 3 & 4 & 5 & 6 & 7 & 8 & 9 & 10 & II & 12 \\
\hline \multicolumn{13}{|l|}{ Day 0} \\
\hline Total Lactobacilli & $1 \times 10^{6}$ & $7.5 \times 10^{5}$ & $1.6 \times 10^{5}$ & $1 \times 10^{5}$ & $1 \times 10^{6}$ & $9.3 \times 10^{4}$ & $7.6 \times 10^{7}$ & $5 \times 10^{5}$ & $4 \times 10^{4}$ & $2.6 \times 10^{6}$ & $6.8 \times 10^{6}$ & $1 \times 10^{7}$ \\
\hline L. paracasei-like & $1.3 \times 10^{4}$ & $2.2 \times 10^{6}$ & $0 \times 10^{3}$ & $8.6 \times 10^{3}$ & $3 \times 10^{3}$ & $9 \times 10^{4}$ & $6.8 \times 10^{7}$ & $8 \times 10^{3}$ & $4 \times 10^{3}$ & $2.4 \times 10^{6}$ & $5.5 \times 10^{5}$ & $0 \times 10^{3}$ \\
\hline Bifidobacteria & $8 \times 10^{8}$ & $3.7 \times 10^{8}$ & $0 \times 10^{6}$ & $3.5 \times 10^{9}$ & $9 \times 10^{7}$ & $1.8 \times 10^{8}$ & $1.5 \times 10^{9}$ & $4.6 \times 10^{6}$ & $0 \times 10^{4}$ & $2.2 \times 10^{9}$ & $3.4 \times 10^{7}$ & $1.6 \times 10^{9}$ \\
\hline \multicolumn{13}{|l|}{ Day 5} \\
\hline Total Lactobacilli & $1.2 \times 10^{6}$ & $2.4 \times 10^{7}$ & $2.6 \times 10^{6}$ & $5 \times 10^{7}$ & $9.8 \times 10^{6}$ & $8.5 \times 10^{5}$ & $2.7 \times 10^{8}$ & $9.5 \times 10^{6}$ & $3.3 \times 10^{7}$ & $1.4 \times 10^{8}$ & $3.8 \times 10^{6}$ & $2.8 \times 10^{7}$ \\
\hline L. paracasei-like & $1.5 \times 10^{6}$ & $2.3 \times 10^{7}$ & $2.1 \times 10^{6}$ & $3.9 \times 10^{7}$ & $9.5 \times 10^{6}$ & $8.3 \times 10^{5}$ & $2 \times 10^{8}$ & $9.5 \times 10^{6}$ & $2.7 \times 10^{7}$ & $1.4 \times 10^{8}$ & $3.4 \times 10^{6}$ & $1.7 \times 10^{7}$ \\
\hline Bifidobacteria & $7.7 \times 10^{8}$ & $4.2 \times 10^{8}$ & $1.5 \times 10^{9}$ & $4.5 \times 10^{7}$ & $3.4 \times 10^{6}$ & $3 \times 10^{7}$ & $2.4 \times 10^{8}$ & $4.3 \times 10^{8}$ & $2.6 \times 10^{7}$ & $2.4 \times 10^{8}$ & $3.7 \times 10^{6}$ & $4.2 \times 10^{9}$ \\
\hline \multicolumn{13}{|l|}{ Day 10} \\
\hline Total Lactobacilli & $4 \times 10^{5}$ & $2 \times 10^{8}$ & $8.3 \times 10^{6}$ & $2.8 \times 10^{7}$ & $3.2 \times 10^{6}$ & $2.2 \times 10^{6}$ & $4.1 \times 10^{8}$ & $1.6 \times 10^{7}$ & $8.6 \times 10^{7}$ & $9.7 \times 10^{5}$ & $7.5 \times 10^{6}$ & $7.6 \times 10^{6}$ \\
\hline L. paracasei-like & $1.7 \times 10^{5}$ & $1.1 \times 10^{8}$ & $6.5 \times 10^{6}$ & $2.8 \times 10^{7}$ & $2.8 \times 10^{6}$ & $2.1 \times 10^{6}$ & $3.5 \times 10^{8}$ & $1.5 \times 10^{7}$ & $1.7 \times 10^{7}$ & $9.4 \times 10^{7}$ & $1 \times 10^{6}$ & $1 \times 10^{6}$ \\
\hline Bifidobacteria & $9.2 \times 10^{8}$ & $1.4 \times 10^{10}$ & $2.3 \times 10^{7}$ & $2.6 \times 10^{9}$ & $2.4 \times 10^{6}$ & $2.3 \times 10^{8}$ & $7.5 \times 10^{8}$ & $3.4 \times 10^{9}$ & $4.3 \times 10^{7}$ & $2.3 \times 10^{7}$ & $3.9 \times 10^{7}$ & $2.5 \times 10^{9}$ \\
\hline \multicolumn{13}{|l|}{ Day 15} \\
\hline Total Lactobacilli & $6 \times 10^{6}$ & $3.7 \times 10^{8}$ & $9 \times 10^{6}$ & $2.5 \times 10^{7}$ & $1.5 \times 10^{7}$ & $2.8 \times 10^{6}$ & $4.4 \times 10^{8}$ & $5.9 \times 10^{8}$ & $3 \times 10^{7}$ & $1.5 \times 10^{8}$ & $5.7 \times 10^{5}$ & $4.1 \times 10^{6}$ \\
\hline L. paracasei-like & $5.7 \times 10^{6}$ & $4.2 \times 10^{8}$ & $9 \times 10^{6}$ & $2.2 \times 10^{7}$ & $1.3 \times 10^{7}$ & $2.8 \times 10^{6}$ & $3.7 \times 10^{8}$ & $2 \times 10^{7}$ & $2 \times 10^{7}$ & $1.1 \times 10^{8}$ & $1.5 \times 10^{5}$ & $4.1 \times 10^{6}$ \\
\hline Bifidobacteria & $4.6 \times 10^{9}$ & $1.4 \times 10^{10}$ & $7.2 \times 10^{8}$ & $3.1 \times 10^{9}$ & $2.3 \times 10^{7}$ & $5.5 \times 10^{9}$ & $1.3 \times 10^{10}$ & $2.9 \times 10^{8}$ & $3 \times 10^{7}$ & $3.5 \times 10^{9}$ & $2.6 \times 10^{8}$ & $2.6 \times 10^{7}$ \\
\hline
\end{tabular}

Table 2: Viable bacterial counts from faecal samples at day 3 of post-treatment period. Individual values. Values are in log of CFU/g of faeces.

\begin{tabular}{lllllllllllll}
\hline Volunteer N. & I & 2 & 3 & 4 & 5 & 6 & 7 & 8 & 9 & 10 & 11 & 12 \\
\hline Total Lactobacilli & $8.1 \times 10^{6}$ & $7.8 \times 10^{8}$ & $9.5 \times 10^{7}$ & $8.8 \times 10^{5}$ & $6.5 \times 10^{6}$ & $1.5 \times 10^{4}$ & $1.2 \times 10^{7}$ & $1.4 \times 10^{7}$ & $3 \times 10^{5}$ & $2.1 \times 10^{9}$ & $2.3 \times 10^{8}$ & $3.8 \times 10^{5}$ \\
L. paracasei-like & $1.5 \times 10^{6}$ & $4.8 \times 10^{8}$ & $1.9 \times 10^{6}$ & $8.5 \times 10^{4}$ & $2.8 \times 10^{5}$ & $1.5 \times 10^{4}$ & $1.1 \times 10^{7}$ & $1.2 \times 10^{7}$ & $1.5 \times 10^{5}$ & $6.4 \times 10^{6}$ & $2.3 \times 10^{6}$ & $3.1 \times 10^{4}$ \\
Bifidobacteria & $2 \times 10^{9}$ & $3.6 \times 10^{9}$ & $5.2 \times 10^{8}$ & $3.1 \times 10^{9}$ & $6.5 \times 10^{8}$ & $0 \times 10^{5}$ & $2.3 \times 10^{7}$ & $1 \times 10^{7}$ & $4 \times 10^{5}$ & $6.4 \times 10^{9}$ & $3.8 \times 10^{8}$ & $4.4 \times 10^{9}$
\end{tabular}

In the post administration period, the counts of $L$. paracasei group in 7 subjects were similar to those achieved at the end of treatment whereas in 5 subjects a decrease of more than one log was found. At the same timepoint, only 3 subjects had low counts $\left(<10^{5}\right)$ of $L$. paracasei group compared to 7 subjects in the baseline period (Table 2).

In 8 out of 12 subjects, an increase of at least one log was observed in the counts of bifidobacteria at end of treatment compared to the baseline sample. In half of the subjects an increase of $3 \log$ was observed. Similarly, an increase of total lactobacilli was found in 9 subjects at end of treatment (Table 1).

No consistent changes were found in the counts of Enterobacteriacee, enterococci and Clostridium during the study.

\section{Genetic identification of L. paracasei}

Genetic analysis was carried out to identify and quantify isolates $L$. paracasei really belonging to this species among all the vancomicin insensitive CFU of lactobacilli. Results actually showed that most of them were L. paracasei (Fig. 1). In fact 60 out of 65 (92\%) CFU at 10-day, 49 out of 58 (84\%) CFU at 15-day and 105 out of 132 (80\%) vancomicin CFU at 3-day post-administration were $L$. paracasei (Table 3).

\section{Discussion}

A novel synbiotic preparation has been assessed by means of an in vivo nutritional trial. Quite surprisingly, only two papers are available on the assessment of the efficacy of synbiotic products $[22,23]$ and none of them have used genetic tools to monitor the fate of the probiotic bacteria.

Strains used in this work have been carefully selected by the most commonly used in vitro tests for the study of probiotic strains [24]. Three strains, L. paracasei B21060, B 21070 and L. gasseri B21190 have finally been selected. These strains have shown a resistance to gastric acidity equal or superior to the reference strains. Similar results were obtained in bile acid resistance tests.

Adhesion to human epithelial cells (buccal cells and intestinal cells) was previously assessed [24] and shows that adhesion is more pronounced for B21060 and 21070 than for B21190 and reference strains (ATCC 53103 and ATCC 23850).

Following the screening period, a combination of the selected probiotics were included in a synbiotic preparation with oligosaccharides, glutamin, vitamin B6 and zinc. 


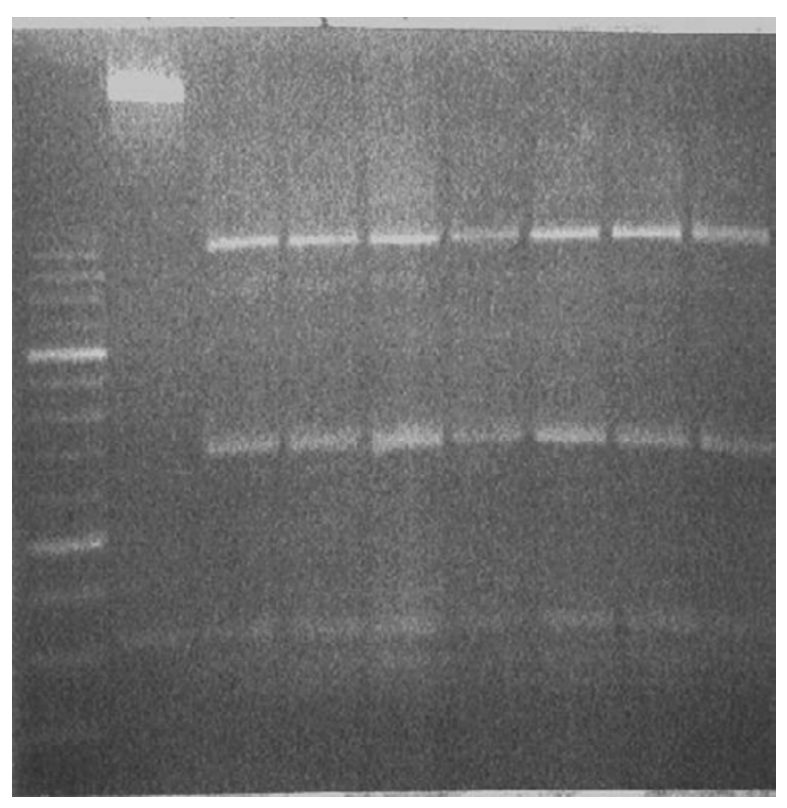

\section{$\begin{array}{lllllllll}1 & 2 & 3 & 4 & 5 & 6 & 7 & 8 & 9\end{array}$}

\section{Figure I}

ARDRA analysis from the left: Lane I. Molecular Weight Marker (Roche)Lanes 2: CFU not identified as $L$. paracasei Lanes 3 to 8: CFUs identified as L. paracasei Lane 9: ARDRA of the reference strain DSM $5622^{\top}$

Table 3: ARDRA identification of $L$. paracasei among the vancomycin insensitive lactobacilli. Values are expresses as positive identification/CFU analysed.

\begin{tabular}{lccc}
\hline \multicolumn{3}{c}{ Synbiotic Intake } & Post-treatment \\
\hline Vol. no. & Day I0 & Day I5 & Day +3 \\
\hline 1 & $3 / 4$ & $5 / 5$ & $9 / 9$ \\
2 & $6 / 8$ & $10 / 18$ & $4 / 6$ \\
3 & $3 / 3$ & $0 / 0$ & $3 / 3$ \\
4 & $3 / 3$ & $5 / 5$ & $9 / 9$ \\
5 & $5 / 5$ & $9 / 9$ & $16 / 18$ \\
6 & $7 / 7$ & $3 / 3$ & $2 / 2$ \\
7 & $5 / 7$ & $1 / 2$ & $7 / 10$ \\
8 & $20 / 20$ & $2 / 2$ & $6 / 6$ \\
9 & $4 / 4$ & $2 / 2$ & $11 / 11$ \\
10 & $2 / 2$ & $9 / 9$ & $33 / 33$ \\
11 & $2 / 2$ & $2 / 2$ & $3 / 23$ \\
12 & $0 / 0$ & $1 / 1$ & $2 / 2$ \\
\hline
\end{tabular}

In the present study, the ability of the probiotics strains to survive through intestinal transit and persist after discontinuation of intake was investigated in healthy volunteers. An increase of faecal counts of vancomycin insensitive group was consistently observed; in fact an increase of at least one $\log$ in the counts of $L$. paracasei-like was found in all subjects. A marked increase ( $>2-3 \operatorname{logs})$ was found in subjects who had low counts of this group of lactobacilli at baseline suggesting that the rate of growth of the administered strains is even greater in subjects who have reduced counts of this species and may be more exposed to the adverse consequences of ecological imbalance.

The above change was already apparent after 5 days of intake which suggests that potential probiotic benefits can be obtained after only few days of intake.

Analysis of faecal samples after 3 days in the post-treatment period shows that strains tend to persist as the counts of $L$. paracasei group (which includes the administered strains) are similar or only slightly decreased compared to those achieved at the end of treatment and higher than those observed at baseline.

Genotypic analysis confirmed that increase of strains phenotypically resembling $L$. paracasei group is related to actual increase of $L$. paracasei species.

With regard to the effects on other intestinal bacteria, the intake of the synbiotic preparation was accompanied by an increase of bifidobacteria. This beneficial effect could be related to the presence of oligosaccharide in the formulation. No consistent effects were seen in other indigenous bacteria, at least in those plate counted.

The tolerability of the preparation was excellent in all individuals. No gastrointestinal or systemic adverse effects were observed during the study

\section{Conclusions}

In this study we have shown that new human indigenous probiotic strains of $L$. paracase $i$ administered in a synbiotic preparation can be recovered from the faeces of healthy human volunteers and rapidly become the numerically dominant Lactobacillus isolated in faecal samples. These strains seems to persist in the colon for at least 3 days after discontinuation of the oral intake. During the study a favourable increase in total lactobacilli and bifidobacteria was also found.

These results are very promising since the study on faecal samples may underestimate colonization of colonic mucosa by probiotic strains even if in the present study microbiological analysis were made on fresh faecal samples. However the results of this study should be con- 
firmed by further studies designed to determine the minimum and optimal dose to achieve effective counts on the colonic mucosa and including a more prolonged period of observation to evaluate the actual duration of persistence of these strains in the large intestine.

\section{Competing interests}

Costs of the trial were funded by Bracco SpA.

\section{Authors' contributions}

Lorenzo Morelli, Daniela Zonenschain, and Maria Luisa Callegari have been in charge of the microbiological and genetic analysis.

Enzo Grossi, Federico Maisano and Michele Fusillo were responsible of the trial management.

\section{References}

I. GL Simon and SL Gorbach: Intestinal flora in health and disease. Gastroenterol 1984, 86:174-93.

2. S Bengmark: Ecological control of the gastrointestinal tract. The role of the probiotic flora. Gut 1998, 42:2-7.

3. McCracken VJ and Lorenz RG: The gastrointestinal ecosystem: a precarious alliance among epithelium, immunity and microbiota. Cell Microbiol 200I, 3: I-II.

4. Fabia R, Ae'Rajab A and Johansson ML et al:: Impairment of bacterial flora in human ulcerative colitis and experimental colitis in the rat. Digestion 1993, 54:248-55.

5. Bengmark S: Econutrition and health maintenance. A new concept to prevent GI inflammation, ulceration and sepsis. Clin Nutr 1996, I5: I-I0.

6. Rusuler-van Embden JGH, Schouten WR and van Lieshout LMC: Pouchitis: result of microbial imbalance? Gut 1994, 35:658-64.

7. Fuller R: Probiotics in human medicines. Gut I991, 32:439-42.

8. MacFarlane GT and Cummings JH: Probiotics and prebiotics: can regulating the activities of intestinal bacteria benefit health? BM] 1999, 3 I 8:999-1003.

9. Gibson GR and Roberfroid MB: Dietary modulation of the human colonic microbiota: introducing the concept of prebiotics. J Nutr 1995, I 25: | 40|-12.

10. Andersson H, Asp N-G, Bruce A, Roos S, Wadstrom T and Wold Ae: Health effects of probiotics and prebiotics: a literature review on human studies. Scand J Nutr 200I, 45:58-75.

II. Majamaa H, Isolauri E, Saxelin M and Vesikari T: Lactic acid bacteria in the treatment of acute rotavirus gastroenteritis. J Pediatr Gastroent Nutr 1995, 20:333-8.

12. Gionchetti, Rizzello $\mathrm{F}$ and Venturi et al.: Oral bacteriotherapy as maintenance treatment in patients with chronic pouchitis: a double-blind placebo controlled trial. Gastroenterol 2000, 19:305-9.

13. Gorbach SL, Chang TW and Goldin B: Successful treatment of relapsing clostridium difficile colitis with Lactobacillus GG. Lancet 1987, 26:1519.

14. Elmer GW, Surawicz CM and McFarland LV: Biotherapeutic agents. A neglected modality for the treatment and prevention of selected intestinal and vaginal infections. JAMA 1996, 75:870-76.

15. Gill HS, Cross ML, Rutherfurd KJ and Gopal PK: Dietary probiotic supplementation to enhance cellular immunity in the elderly. Br J Biomed Sci 200I, 57:94-6.

16. Food Agriculture Organization: Guidelines for the evaluation of probiotics in food. [http://www.agr.gc.ca/food/nff/pdfdocs/probiot ics/pdf].

17. Collins JK, Thronton G and O'Sullivan GO: Selection of probiotic strains for human applications. Int Dairy J 1998, 8:487-90.

18. Van Loo J, Cummings J, Delzenne N, Englyst H, Franck A, Hopkins M, Kok N, Macfarlane G, Newton D, Quigley M, Roberfroid M, van Vliet $T$ and van den Heuvel E: Functional food properties of nondigestible oligosaccharides: a consensus report from the
ENDO project (DGXII AIRII-CT94-1095). Br 」 Nutr 1999, 2:121-32.

19. Rastall RA and Maitin V: Prebiotics and synbiotics: towards the next generation. Curr Opin Biotechnol 2002, 13:490-6.

20. Reniero R, Morelli L, De Haen C and Bottazzi V: Detection of permanent Lactobacillus casei subsp. strains in weaned infants' gut. Lett Appl Microbiol I99I, 13:3-6.

21. Ventura M, Casas IA, Morelli $L$ and Callegari ML: Rapid amplified ribosomal DNA restriction analysis (ARDRA) identification of Lactobacillus spp. Isolated from faecal and vaginal samples. System Appl Microbiol 2000, 23:504-9.

22. Kanamori $Y$, Hashizume K, Sugiyama M, Mortomi M, Yuki N and Tanaka $R$ : A novel synbiotic therapy dramatically improved the intestinal function of a pediatric patient with laryngotracheo-esophageal cleft (LTEC) in the intensive care unit. Clin Nutr 2002, $21: 527-30$.

23. Gmeiner M, Kneifel W, Kulbe KD, Wouters R, De Boever P, Nollet $L$ and Verstraete $W$ : Influence of a synbiotic mixture consisting of Lactobacillus acidophilus 74-2 and a fructooligosaccharide preparation on the microbial ecology sustained in a simulation of the human intestinal microbial ecosystem (SHIME reactor). Appl Microbiol Biotechnol 2000, 53:219-23.

24. Drago L, Gismondo MR, Lombardi A, de Haen C and Gozzini L: Inhibition of in vitro growth of enteropathogens by new Lactobacillus isolates of human intestinal origin. FEMS Microbiol Lett 1997, 1 53:455-63.
Publish with Bio Med Central and every scientist can read your work free of charge

"BioMed Central will be the most significant development for disseminating the results of biomedical research in our lifetime. "

Sir Paul Nurse, Cancer Research UK

Your research papers will be:

- available free of charge to the entire biomedical community

- peer reviewed and published immediately upon acceptance

- cited in PubMed and archived on PubMed Central

- yours - you keep the copyright

Submit your manuscript here:

http://www.biomedcentral.com/info/publishing_adv.asp
BiolMedcentral 